\title{
Des rapports à l'école à Djibouti : entre confiance et défiance
}

Relationships to school in Djibouti: Between trust and distrust

Acerca de las relaciones en la escuela en Yibuti: entre confianza y desconfianza

\section{Rachel Solomon Tsehaye}

\section{(2) OpenEdition}

Journals

Édition électronique

URL : https://journals.openedition.org/ries/5518

DOI : 10.4000/ries.5518

ISSN : 2261-4265

Éditeur

France Education international

Édition imprimée

Date de publication : 1 septembre 2016

Pagination : 91-102

ISBN : 978-2-85420-611-1

ISSN : $1254-4590$

\section{Référence électronique}

Rachel Solomon Tsehaye, «Des rapports à l'école à Djibouti : entre confiance et défiance », Revue internationale d'éducation de Sèvres [En ligne], 72 I septembre 2016, mis en ligne le 01 septembre 2018, consulté le 01 juillet 2021. URL : http://journals.openedition.org/ries/5518; DOI : https://doi.org/ $10.4000 /$ ries.5518

(c) Tous droits réservés 


\section{Des rapports à l'école à Djibouti : entre confiance et défiance}

\section{Rachel Solomon Tsehaye \\ Université de Fribourg}

L'éducation, à travers son outil privilégié qu'est l'institution scolaire, permet à la société d'exercer un contrôle sur les nouvelles générations (Foucault, 1976). Derrière l'autorité des enseignants se dissimulent des exigences sociales (Foray, 2009). L'institution scolaire renvoie à une délégation de la responsabilité éducative, les enseignants agissant au nom de la société et en vue de pérenniser les traits caractéristiques qui lui assurent une cohésion. Sans omettre les fonctions économique et démographique, dont l'importance varie en fonction des contextes, l'institutionnalisation de l'éducation répond à une fonction idéologique de socialisation. En effet, quel que soit le contexte, les questions du contrôle de la population et de la diffusion des normes ont été résolues à travers un mode d'imposition privilégié : l'école, ses dispositifs et ses contenus d'enseignements. C'est autour de cette fonction idéologique « d'imposition d'un arbitraire culturel " (Bourdieu et Passeron, 1970) que la question de l'autorité sera revisitée et que le régime de légitimité, entendu comme un "régime de justification rationnelle" (Cornu, 2009) des écoles en présence dans le contexte djiboutien (école coranique, madrasa, école privée catholique et école publique laïque) sera examiné.

\section{CONTEXTE DE SCOLARISATION ET PROBLÉMATIQUE}

Le contexte de scolarisation à Djibouti se caractérise d'une part par les écoles coraniques, qui incarnent la généralisation d'un enseignement public et religieux pour tous. Les enseignements se déroulent dans la rue : filles et garçons apprennent par cœur le Coran par la voie de la récitation orale et de l'écriture à l'encre sur planche de bois. La madrasa constitue le niveau secondaire de l'enseignement coranique : l'enseignement y est non-mixte. Elle intègre l'enseignement réflexif de l'islam en langue arabe et d'autres disciplines, telles que l'arithmétique, la poésie, la langue et la littérature arabes. Ces deux écoles renvoient au "système-monde " ${ }^{1}$ moyen-oriental, lequel promeut une conception d'origine religieuse du monde et un ensemble de normes (linguistique, savoirs locaux, etc.) qui régissent le vivre-ensemble à Djibouti.

1. Cette expression est empruntée à Immanuel Wallerstein, qui a développé le concept ; elle est tirée de la notion d'« économie-monde », dont Fernand Braudel est l'inventeur. 
Dans le système-monde (SM) occidental figurent les écoles modernes, publique et laïque d'une part, privée catholique de l'autre. Dans ces écoles, les curricula dispensés en langue française, sont analogues à ceux établis en France, connaissant un émiettement disciplinaire identique, mais avec quelques adaptations (l'arabe en première langue vivante). L'héritage religieux y est mis entre parenthèse (l'école catholique accueille $99 \%$ de musulmans), et l'islam dogmatique est contrarié par l'individualisme moderne, le matérialisme inhérent à l'industrialisation et le paradigme scientifique qui suppose d'avancer dans l'incertitude, de douter et d'avoir confiance en la raison critique.

Se confrontent ainsi la promotion de l'autonomie individuelle, qui est au centre des enseignements implicites des écoles du système-monde occidental, et celle de l'apprentissage du lien social et de l'appartenance à la communauté musulmane (l'Umma), en cours dans les écoles musulmanes. Se distinguent également des savoirs locaux, religieux et coutumiers, les savoirs scolaires dont les fondements ont été érigés suite à une rupture avec la religion, et qui relèvent d'un type spécifique de savoir - scientifique. Enfin, la distribution des élèves dans les écoles du système-monde oriental suit une logique d'éducation mutuelle non certificative, l'école étant « un lieu d'entraînement du corps et de l'esprit à l'endurance physique et morale » (Diop, 1960) poursuivant l'objectif de l'intégration morale et culturelle de l'enfant dans la société locale. Celle-ci se distingue de la logique de distribution / hiérarchisation verticale des élèves dans le systèmemonde occidental, dans lequel la socialisation vise à former des individus compétents, malléables, flexibles pouvant répondre à des exigences fonctionnelles.

Les écoles du système-monde occidental, descendantes de l'école coloniale tenue par les religieux (catholiques), bénéficient d'une double légitimité, malgré diverses caractéristiques exogènes (savoirs scolaires, langue d'enseignement, intentions civilisatrices, etc.) : leur autorité s'est construite à travers le processus de colonisation, avant d'être consolidée par l'internationalisation de l'éducation. Elles promeuvent un ensemble de normes à travers un curriculum ouvert à la citoyenneté mondiale, par lequel les savoirs scolaires fondés sur le paradigme scientifique se voient officiellement asseoir et sédimentent leur autorité. Elles privilégient un système-monde (éducation occidentale de type laïque, individualiste, universaliste aux savoirs modernes et scientifiques) par rapport à l'autre (religieux, communautaire, particulariste aux savoirs traditionnels et religieux). Or «les théories ne sont pas neutres " (Cornu, 2009) et peuvent potentiellement générer un conflit d'autorité entre les finalités éducatives des écoles des deux systèmes-mondes.

\section{QUESTIONS DE RECHERCHE ET MÉTHODE}

Les enjeux de la recherche sont caractérisés par l'asymétrie de pouvoir entre les savoirs que révèlent les curricula de l'école officielle, donc le parti pris (ici laïque) de l'État pour la société (ici musulmane). Ils supposent de s’interroger 
sur la valeur attachée aux savoirs scientifiques, tenus pour légitimes dans l'espace scolaire et toujours plus renforcés par les politiques éducatives mondiales. L'objectif est de retirer des discours des acteurs ce qui éclaire les rapports à l'école et révèle leur nature, tout en renseignant sur la confiance et/ou la défiance suscitées par chacun des modèles éducatifs. Ces relations de confiance seront étudiées au prisme des expériences scolaires antérieures, des projets parentaux pour leurs enfants, si ce n'est des projets d'avenir pour les plus jeunes, relatés dans les entretiens.

Les présentes analyses sont tirés de 72 entretiens semi-directifs décomposés comme suit : 14 parents d'élèves (P), 14 instituteurs d'écoles primaires (IP), 7 instituteurs de madrasa (IM), 8 maîtres d'école coranique (MC), 8 directeurs/rices d'écoles publiques (DP), 6 directeurs de madrasa (DM) et 15 jeunes déscolarisés (JD). Le protocole comprenait également des observations non-participantes du quotidien scolaire, dans les quatre types d'écoles concernées par la recherche.

\section{Typologie des rapports à l'école}

Les grandes tendances mises au jour par le traitement des résultats révèlent trois types de rapports à l'école. Ils sont utilisés ici comme un outil permettant de placer la confiance (et son image inversée, la défiance, considérée comme absence de crédit) au centre de la réflexion. Leur examen a été rendu possible par des questions gravitant autour de la confiance en l'institution, de l'autorité des savoirs, de l'autorité de l'enseignant et des expériences subjectives de scolarisation.

\section{LE RAPPORT À L'ÉCOLE INDIVIDUALISTE, CONFORMISTE, PRAGMATIQUE}

\section{De la confiance vis-à-vis des savoirs scolaires...}

Les acteurs qui scolarisent leurs enfants à l'école publique ou, pour les plus favorisés d'entre eux sur le plan économique, à l'école privée catholique (Solomon Tsehaye, 2014) témoignent d'une confiance absolue vis-à-vis des savoirs scolaires, les seuls qui fassent autorité à leurs yeux. Ces individus témoignent d'une représentation positive de l'institution scolaire, leur progéniture étant engagée pour la plupart dans des parcours non problématiques (parents francophones, familiarité avec l'écrit, discernement et explicitation de l'implicite). Valorisant tout savoir participant d'un curriculum permettant de bénéficier des bienfaits de la mondialisation (obtention d'un emploi salarié moderne), le curriculum proposé par les écoles coraniques et les madrasa se trouve de fait « inutile ", 
Tableau 1. Entre confiance et défiance : typologie des rapports à l'école

\begin{tabular}{|c|c|c|c|c|c|}
\hline \multirow[b]{2}{*}{$\begin{array}{l}\text { Rapports } \\
\text { à l'école }\end{array}$} & \multirow[b]{2}{*}{$\begin{array}{l}\text { Finalités } \\
\text { de l'école }\end{array}$} & \multicolumn{2}{|c|}{$\begin{array}{l}\text { Identification } \\
\text { au SM occidental }\end{array}$} & \multicolumn{2}{|c|}{$\begin{array}{l}\text { Identification } \\
\text { au SM oriental }\end{array}$} \\
\hline & & $\begin{array}{l}\text { Conception } \\
\text { de la vie } \\
\text { orientée } \\
\text { sur le travail } \\
\text { productif }\end{array}$ & $\begin{array}{l}\text { Convergence } \\
\text { des réseaux } \\
\text { autour } \\
\text { de l'école - } \\
\text { communauté } \\
\text { internationale }\end{array}$ & $\begin{array}{l}\text { Conception } \\
\text { de la vie } \\
\text { orientée } \\
\text { autour } \\
\text { de Dieu }\end{array}$ & $\begin{array}{c}\text { Convergence } \\
\text { des réseaux } \\
\text { autour } \\
\text { de la } \\
\text { communauté } \\
\text { locale }\end{array}$ \\
\hline $\begin{array}{l}\text { Individualiste } \\
\text { conformiste } \\
\text { pragmatique }\end{array}$ & $\begin{array}{l}\text { un emploi salarié } \\
\text { moderne - } \\
\text { intégration } \\
\text { au niveau } \\
\text { international }\end{array}$ & forte & forte & faible & faible \\
\hline $\begin{array}{l}\text { Replié } \\
\text { collectiviste } \\
\text { idéaliste }\end{array}$ & $\begin{array}{l}\text { être un « bon } \\
\text { musulman » - } \\
\text { intégration } \\
\text { sociale } \\
\text { au niveau local }\end{array}$ & faible & faible & forte & forte \\
\hline $\begin{array}{l}\text { Conciliatoire } \\
\text { complémentaire } \\
\text { cosmopolite }\end{array}$ & $\begin{array}{c}\text { des connaissances } \\
\text { une instruction } \\
\text { servant la société } \\
\text { comme } \\
\text { l'épanouissement } \\
\text { de soi }\end{array}$ & $\begin{array}{c}\text { faible } \\
\text { ou forte }\end{array}$ & $\begin{array}{c}\text { forte } \\
\text { ou faible }\end{array}$ & $\begin{array}{c}\text { faible } \\
\text { ou forte }\end{array}$ & $\begin{array}{c}\text { faible } \\
\text { ou forte }\end{array}$ \\
\hline
\end{tabular}

Source : réalisé par l'auteure.

puisqu'il ne sert pas leur projet éducatif (P8). Les écoles coraniques et les madrasa sont considérées comme des outils aux mains d'un État autoritaire, qui ne concourent ni à l'augmentation des taux de scolarisation dans l'enseignement public ni à enrayer l'obscurantisme (P9, IP17). À leurs yeux, l'éducation de type occidental constitue la voie royale au service de l'intégration dans le secteur économique formel, pour laquelle l'obtention d'un diplôme, qui plus est « reconnu » à l'échelle internationale, est indispensable.

\section{...à la défiance vis-à-vis de l'éducation musulmane et des savoirs locaux}

Le processus identificatoire aux normes et pratiques normatives véhiculées par le système-monde occidental (défense de la liberté individuelle, croyances dans les savoirs scientifiques, usage de la langue française, etc.) mène certains acteurs à se réclamer de la «culture française » (IP4, P10) et à s'exclure de la communauté locale : ainsi IP8 comparait négativement les madrasa à des "écoles d'indigènes ". Cette affection idéalisante peut renvoyer certains à une nostalgie des temps coloniaux :

"C'était bien quand les Français nous éduquaient, ils enseignaient bien car c'était leur langue !!» (P1) 
Autorité par confiance accordée à/au(x)

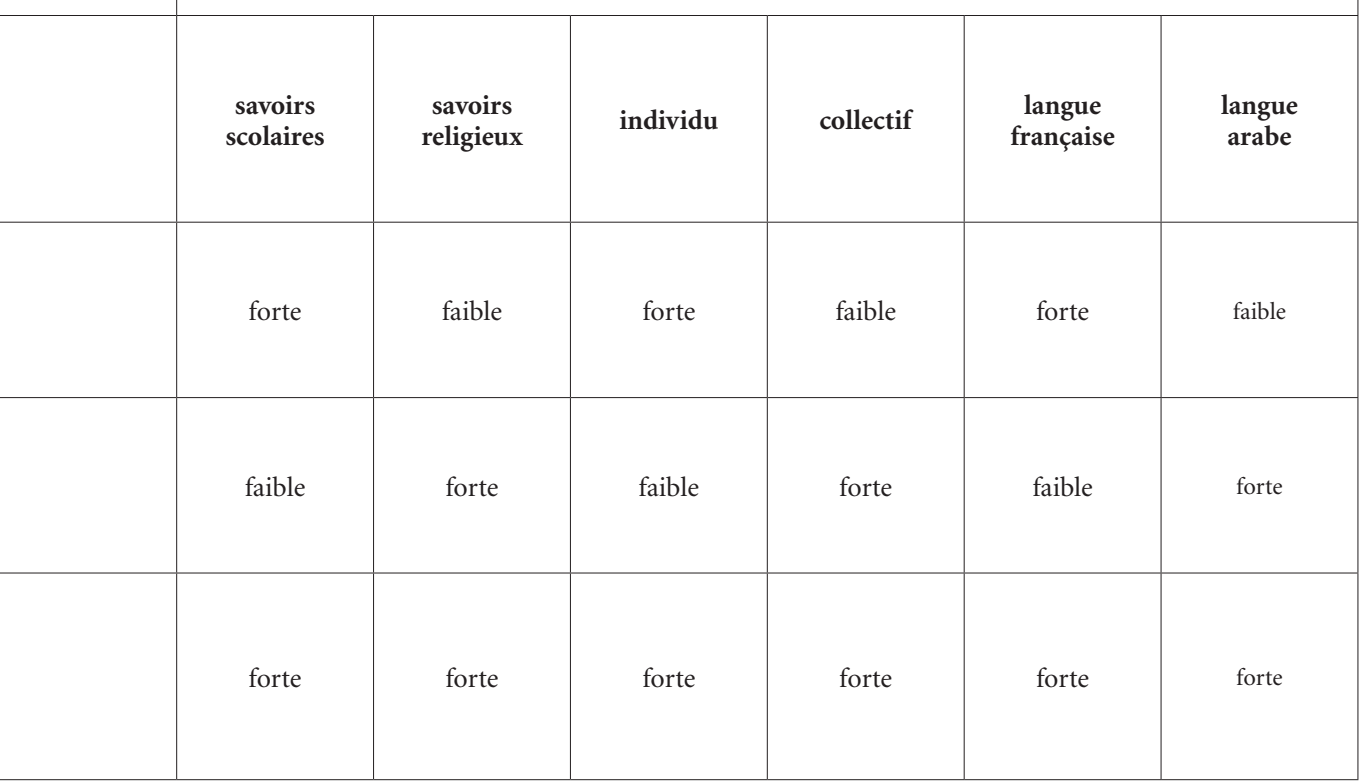

Comparaison sociale oblige, s'échappe également des discours un sentiment de bénéficier, depuis, d'un enseignement au rabais : "à l'époque coloniale, quand même l'école était mieux!»(DP2). Ce regret tient à la dégradation des conditions d'enseignement et d'apprentissage (hausse des effectifs et baisse des moyens) mais aussi, comme le rappelle IP12, " au jeune âge du pays qui [avait en 2004] à peine 26 années d'indépendance après 120 ans de colonisation ». Cette posture identificatoire entraîne un mécanisme de dévalorisation des savoirs locaux :

«Si les enseignants parlent en langue locale, ce n'est pas comme ça que l'enfant va faire un effort. Quand les enseignants parlent en somali, je dis madame arrête ça, parle-lui en français. » (DP6).

Les langues locales sont dépréciées par ces acteurs, au point de paraître indignes de respect (DP4), et de figurer comme de potentiels dangers pouvant faire courir le risque de "dérapage ethnique " à la société (DP7). Ce rapport entraîne également une radicalisation de la posture occupée à l'égard de l'école coranique, qui servirait l'avilissement des individus, à travers l'apprentissage de la violence physique, qui la rend totalement illégitime sur le plan pédagogique :

«Les enfants acceptent d'être frappés par le maître, ils ne se révoltent pas, ils acceptent cette soumission! Ça vient de l'école coranique, parce qu'ils étaient éduqués comme ça! Cette soumission, c'est incroyable... !» (DP2). 
Ce discours contribue au sentiment de désaveu, de perte de légitimité et de discréditation des maîtres coraniques et des enseignants de madrasa, par la remise en question de leurs compétences éducatives. Il reflète également une représentation particulière de l'islam, qui est posé en image inversée de la modernité, intolérant et fanatique.

\section{...à la défiance vis-à-vis des pratiques éducatives enseignantes}

La confiance absolue dans les savoirs scolaires est juxtaposée à une confiance relative en l'autorité que figurent les enseignants. La confiance est alors mise à mal à travers des expériences négatives. Ainsi, si les parents sont fermement attachés à l'idée que leurs enfants s'approprient un curriculum ouvert sur la modernité et l'international, ils déplorent les châtiments corporels que leurs enfants subissent en classe. Leur discours évoque fréquemment le recours à l'autoritarisme comme mode de conduite de classe et d'incarnation de l'autorité par les enseignants du public : "les pratiques pédagogiques ne se sont pas modernisées » (P9), « les enseignants n'ont pas intégré tout ce que l'on sait maintenant sur l'enfance» (P1). Ce que certains appellent ironiquement le «tuyau pédagogique " (IP7, IP9), fait partie de la scénographie du quotidien scolaire dans l'enseignement public (l'interdiction des châtiments corporels étant respectée dans l'enseignement privé catholique). Ce symbole de l'imaginaire du pouvoir induit un rapport de défiance vis-à-vis des pratiques éducatives des enseignants, sans toutefois obérer la confiance portée aux savoirs scolaires.

Certains directeurs d'école publique évoquent « les accrochages avec les parents [qui] portent essentiellement sur les châtiments corporels (...) : on essaie d'arranger, mais bon il y a des gens qui ne passent pas sans tuyau...» (DP2). Ce discours laisse transparaître la confusion entre l'autorité et l'abus d'autorité, qui caractérisait les pratiques éducatives aux temps coloniaux :

" On fumait en cachette, et quand on voyait le directeur d'école ou l'instituteur, on éteignait nos cigarettes sur notre corps, tellement qu'on avait peur, tellement qu'il y avait un respect. » (DP5)

Ce dernier point révèle cette fois la confusion entre la peur et le respect, entre une autorité éducative rationnelle et responsabilisante et une autorité personnifiée qui fonde son pouvoir sur un statut requérant l'obéissance par l'usage de la force et de la contrainte. D'autre part, l'évolution du rapport à l'enfance se voit justement imputé la responsabilité par les enseignants et les directeurs d'école d'être à l'origine d'un «laxisme ambiant» (IP8, DP3, DP2). Le courant est «difficile entre parents et enseignants » (IP7), «l'enfant ne fait pas les devoirs à la maison, bon, ils s'en foutent les parents » (IP9), « ils envoient leurs enfants à l'école pour ne pas être dérangés » (IP7). La défiance mutuelle entre les acteurs d'un même système éducatif ne concerne pas que la relation 
école / famille. La défiance est aussi hiérarchique et verticale : les directeurs d'école interrogés ont tous fait mention du "désengagement des enseignants » (DP4), de leur " difficulté à les motiver »(DP3), au regard des conditions de travail (grands effectifs, double flux, conditions salariales peu favorables car aléatoires). Certains accusent «le personnel [d'être] défaillant»(DP6), d'autres imputent ces difficultés (faibles assiduité, ponctualité et rendement) à des facteurs externes au système (le Khat ${ }^{2}$ notamment).

\section{LE RAPPORT À L'ÉCOLE REPLIÉ, COLLECTIVISTE, IDÉALISTE \\ De la confiance vis-à-vis des savoirs du système-monde oriental...}

La confiance vis-à-vis de l'éducation musulmane se caractérise dans les discours des acteurs par sa "proximité culturelle » avec le système local. L'oralité des causeries dans les mosquées et les madrasa s'apparente aux modes de transmission des savoirs traditionnels : "la madrasa se rapproche plus de nos mœurs»(P7). Aussi, les discours valorisent l'éducation mutuelle :

"J'attends toujours les plus grands avant de commencer car ce sont eux qui vont réciter en chantant les hadiths afin que les petits répètent à haute voix. » (IM2)

Ce sentiment de proximité tient également au vécu individuel et à la généralisation de l'expérience de fréquentation de l'école coranique, ce qui ne vaut pas pour l'école publique (le taux de scolarisation brut dans le primaire avoisine les $52 \%$ en 2008-2012 selon l'UNICEF). Les enfants éduqués dans l'islam sont dits "plus polis, plus hospitaliers, ils sont dans le droit chemin » alors que ceux éduqués dans les écoles de type occidental sont « dans la déchéance, ils fument, ils se sentent libres, émancipés et moqueurs» (DM2). Le fait d'apprendre en madrasa tout un ensemble de préceptes coraniques qui gouvernent la vie quotidienne participe au processus d'affirmation identitaire des acteurs. C'est à ce titre que la ségrégation de genre en place dans les madrasa (alors que la mixité est de mise dans les écoles coraniques) inspire confiance aux parents. Celle-ci leur garantit l'acquisition des interdits communs et des vertus attendues chez les enfants : "la retenue, la réserve, le respect » (IM4), «ne pas fumer, ne pas brouter le Khat, ne pas boire d'alcool, et si c'est une fille, rester voilée »(DM1).

2. Le Khat est une plante qui contient une substance psychotrope. À Djibouti, sa vente et sa consommation sont légalisées et l'objet de rituels quotidiens. 


\section{...à la défiance vis-à-vis de l'école moderne du système-monde occidental}

La défiance à l'égard des écoles du système-monde occidental est avant tout une défiance idéologique, liée à des représentations sociales négatives, fruit des relations coloniales et néo-coloniales, qui jettent la suspicion sur les Occidentaux. Dans les discours, elle conduit les acteurs à ériger et transformer les différences en antagonismes profonds :

«L'école est un podium ou l'on expose ses vêtements provoquants alors que l'éducation musulmane entretient la valeur de "pudeur". » (IM4)

Les savoirs scolaires sont une source de préoccupation car ils propagent l'idéologie du matérialisme et de l'individualisme (M5, P12, IM3) et concourent à délégitimer les institutions traditionnelles. La société occidentale apparaît à leurs yeux comme dangereuse pour l'unité de la famille, ce qui entraîne rejet et résistances à certaines influences. L'école officielle détournerait du respect que la religion est à même d'enseigner (M6) mais surtout des pratiques religieuses :

«Quand ils vont à l'école publique, les parents sont obligés de les frapper pour qu'ils fassent la prière, alors qu'à la madrasa ils deviennent de vrais pratiquants. »(M2)

Il n'est donc pas étonnant qu'il s'agisse « d'écarter les jeunes d'influences néfastes à la culture musulmane fondée sur le respect et l'honneur » (M5). Dans les discours fleurissent également des représentations passéistes idéalisant la figure du maître respecté lorsqu'il symbolisait le seul détenteur et vecteur du savoir scolaire (IP2) et accusant les modalités éducatives de "l'école française » (IP11) d'avoir fait « écrire les enfants pour les faire sortir de notre culture et de notre religion " $(I b$.$) . Enfin, les curricula sont perçus comme menaçants : la raison$ critique est "nocive aux mœurs locales» (DM1), les risques éthiques encourus par le développement scientifique choquent le système de croyances en place:

«Prenons le clonage, pour moi ça dépasse la limite de l'acceptable car on risque le débordement total. Seul Dieu peut créer l'Homme. » (IP6)

La défiance envers les écoles du système-monde occidental et les savoirs scolaires peut également être nourrie par une expérience de disqualification à et par l'école publique. La disqualification tient aux mauvais résultats obtenus aux évaluations, notamment celle du CM2, qui constitue un véritable goulet d'étranglement (il ne reste que $28 \%$ d'entre eux au secondaire, Unicef, 2012). Celle-ci contribue à dégrader l'image de soi et à contester en retour l'autorité qui produit des normes inatteignables. Ainsi, nombre de jeunes déscolarisés ont confié « avoir honte » d'avoir échoué (JD3, JD9, JD10) ou de ne pas savoir parler correctement le français, ce qui fait l'objet d'un dénigrement. L'un d'eux disait à l'autre avant de commencer l'entretien : «Tu sais même pas parler français ! Tu passeras sur 
TV5 et t'auras l'air con!»(JD11). Les jeunes rencontrés imputaient leur échec soit aux mauvaises intentions des enseignants à leur égard, soit à leur propre « nullité » supposée (JD4, JD6...).

Enfin, la défiance se nourrit également des ambitions professionnelles déçues :

Les parents ne voient que ceux qui ont réussi et qui ont eu des bons boulots en français. (DM2)

Ils sont dits "aveuglés par le système »(IP6). Même si certains parents disent scolariser leurs enfants en madrasa "face aux dégâts de l'éducation laïque »(P7), l'autre usage à noter relève d'une stratégie de secours, laquelle concourt à en disqualifier l'enseignement, "à faire de la madrasa une école de la deuxième chance pour ceux qui ne réussissent pas à l'école publique » (DM2). Or, « il y a des jeunes qui ont fait l'école et qui n'ont pas de métier, qui traînent dans les rues»(DM1). La défiance peut donc aussi s'expliquer par l'absence de place pour tous dans la société, et plus particulièrement dans le secteur industriel peu développé et dans un marché de l'emploi salarié moderne qui se résume aux postes dans la fonction publique, laquelle a fermé ses portes suite à des plans d'ajustement structurels. Aussi, le sentiment de disqualification déplacée en madrasa est renforcé par le manque de soutien de l'État: les discours évoquent les inégalités liées aux conditions de traitement salarial, mais aussi le sentiment d'illégitimité liée à leur non-reconnaissance par l'État.

\section{LE RÉGIME CONCILIATEUR, COMPLÉMENTAIRE, COSMOPOLITE De la confiance absolue et générale...}

Ceux qui adoptent un rapport à l'école conciliateur ont une vision éloignée d'un islam féodal, despotique et composent avec le dogme. Ils se rallient à l'analyse historique liant « la langue française à l'entreprise coloniale » (P6) et constatent «qu'elle n'est toujours pas la langue du peuple» (IP10). Des apparentes contradictions et des tensions historiques, ils tissent des stratégies nouvelles : la pratique de double scolarisation ${ }^{3}$ permet de multiplier les chances de réussite scolaire, sociale et professionnelle, et de pouvoir occuper un emploi aussi bien dans l'administration de langue arabe que française, dans les pays arabophones que francophones, car ce sont «les deux langues de travail par lesquelles l'enfant peut gagner sa vie »(IP12). Cette quête des avantages multiples qui entraîne la conciliation des différents modes éducatifs concourt à requalifier les enseignants des madrasa et la madrasa du même coup. La scolarisation en madrasa n'est plus une voie de secours en cas d'échec à l'école publique mais

3. Cf. tableau in Solomon Tsehaye, 2014, p. 92. 
s'inscrit dans la perpétuation de la tradition, les parents considérant que l'exclusion sociale encourue par l'ignorance des savoirs locaux, représente un danger plus important que l'échec à l'école publique. Ainsi, les savoirs locaux ne sont ni discrédités ni même susceptibles de l'être ; ils contribuent à une conception de l'éducation de type holistique. C'est donc en connaissance de cause, parce qu'ils considèrent que leurs enfants auraient plus à perdre qu'à gagner, qu'ils choisissent d'opter pour la double scolarisation. Les acteurs octroient leur confiance, bien que les savoirs scolaires soient renvoyés à leur exogénéité historique : "si tu disais “j”ai appris la conjugaison”, personne ne comprenait » (IP5). Alors que les savoirs scolaires ont été apprivoisés par la plupart de ces parents, ils sont combinés à une pratique religieuse et ne sont pas jugés incompatibles avec l'ordre divin qui régit, selon leurs croyances, la civilisation humaine : ainsi une directrice de l'école publique " prie Dieu pour que (son) fils réussisse à l'école» (DP1). Ces stratégies sont rendues possibles par un processus de double identification et de sentiment d'appartenance aux deux systèmes-mondes, lequel évacue toute pression d'assimilation. L'éducation de type occidental n'exerce pas de menace sur leur identité ; ils ne craignent pas de voir leurs propres valeurs dénigrées ou niées, mais ils composent avec elles une nouvelle voie de normalisation, qui autorise de conjuguer les savoirs scolaires aux savoirs locaux (IP12, MC4...).

\section{...à la confiance relative et sous conditions}

Cependant, la confiance allouée aux écoles des deux systèmes-mondes est conditionnée : l'usage intensif de la contrainte physique comme mode d'exercice de l'autorité suscitent leur désapprobation. Les parents sont conscients des pratiques autoritaires dont ( $\mathrm{ab}$ ) usent les enseignants, aussi bien dans le système public qu'à l'école coranique, pour les avoir eux-mêmes subies. S'ils disposent des moyens économiques suffisants, les parents recourent alors au précepteur particulier (Solomon Tsehaye, op. cit.). Sans quoi, ils s'engagent dans le dialogue et la négociation pour essayer de faire évoluer le modèle conservateur et statutaire de l'autorité :

«Mes enfants vont à l'école coranique mais je leur dis de ne pas les frapper. Même s'il ne vient pas, tu ne les frappes pas, tu me le dis et c'est moi qui m'en occupe. Faut pas les toucher. » (IP14)

Ils relativisent et justifient également l'autoritarisme, en attribuant aux enseignants de bonnes intentions et un fort degré d'exigences :

«Je vois que lui, ce qu'il voulait c'est qu’on travaille. Qu’on réussisse. Mais à ce moment là, on ne comprenait pas pourquoi il était aussi méchant». (P6)

Bien que ces pratiques contrarient le climat de justice et les apprentissages, l'autorité de l'enseignant n'est pas contestée, dans la mesure où l'asymétrie est constitutive de la relation éducative et que d'autres alternatives ne se présentent 
pas. Les enseignants obtiennent l'obéissance des familles malgré le climat d'insécurité car l'école entretient l'espoir tenace de s'extraire de sa condition. Les parents sont conscients du lien de dépendance entre ce type de scolarité et l'avenir professionnel (statut salarié moderne et emploi moderne) et souscrivent donc à l'obligation. Cette obéissance constitue un "acte d'échange " (Reichenbach, 2009) : la confiance n'est pas aveugle mais volontaire, les individus « concédant - au moins implicitement - l'infériorité de leur position » (ib.). En échange de la conformité aux règles du jeu scolaire, le maître assure à l'élève de disposer au bout de longues années de captivité, des clefs de sa propre libération, de son autonomie et de l'assurance d'un avenir sécurisant. Or, à Djibouti, encore moins qu'ailleurs, l'école ne donne pas accès de facto à la réussite et pas plus à l'emploi de type salarié moderne. L'obéissance du présent renvoie à un futur très incertain.

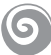

Dans le contexte de la recherche, l'absentéisme n'est pas un symptôme de la crise d'autorité pédagogique, telle qu'elle est observable en Europe, mais il renvoie aux conditions socio-démographiques et économiques, étau dans lequel sont pris les familles et leurs enfants. Si incivilité il y a, elle se situe du côté de l'enseignant plutôt que de l'élève. Si passivité des élèves il y a, elle est liée aux modalités de l'exercice de l'autorité, à l'incompréhension linguistique, à l'impossibilité de mobiliser certaines compétences (familiarité avec l'écrit, ressources lexicales) et catégories de pensée (processus de secondarisation, conscience métalinguistique), qui placent de fait les élèves en situation d'incapacité. Si défiance il y a, elle n'est pas tant liée aux paradigmes scientifique / religieux qui coexistent qu'à la désillusion liée à l'effacement de l'avenir, malgré la captivité consentie (ibidem). Quelle utilité trouver à l'école si celui qui obéit ne tire plus profit de son obéissance ? Comment éviter la perte de confiance liée à l'incompréhension de l'usage qui peut être fait de savoirs scolaires parcellisés et décontextualisés?

Afin que la violence scolaire symbolique ne devienne pas le principal ferment et motif de scolarisation en madrasa, l'urgence est de produire des horizons servant à vaincre le sentiment d'arbitraire, d'efforts vains et d'inutilité, en luttant contre l'autoritarisme contre-productif exercé par l'imposition normative d'un modèle éducatif, la parcellisation des savoirs scolaires et les pratiques en classe. Il s'agirait d'une part d'encourager les transformations sociales et de repenser le travail productiviste, capitaliste et consumériste, et d'interroger la mondialisation, de sorte que ses rentiers actuels puissent envisager d'en partager les bénéfices (soit un avenir digne d'intérêt). Changer le rapport à l'école et donner la possibilité aux élèves de participer concrètement et activement à la construction de l'espace qui les forme apparaît être une initiative utopique (mais non illusoire) et prometteuse : la prise en compte des subjectivités, des 
géographies occupées et des histoires biographiques des individus aurait pour effet vertueux de "passer de la co-errance à la cohérence » (Vieille-Grosjean, 2016) et donc de pallier la "crise du sens» (Kambouchner, 2009). Ce dessein suppose donc d'ouvrir l'école sur la vie sociale et la vie de ses sujets, de rapprocher les savoirs scolaires des savoirs locaux, de reconnaître la distance existante entre les pratiques langagières locales et celles requises par l'école (le français et l'arabe). Cette combinaison donnant à voir la complémentarité des savoirs est une réponse possible au mépris actuel des manières de parler des élèves et de leurs horizons habituels et à l'expurgation des croyances populaires, desquels peut être attendu un détournement du mécontentement populaire sur l'Occident, par un retour en force de l'obscurantisme et des fondamentalismes. L'éducation est un concept qui renvoie à un processus qui s'imagine à plusieurs, par les apprenants et leurs accompagnateurs, avant de se faire.

Souhaitons-nous d'être inventifs.

\section{BibLIOGRAPHIE}

BOURDIEU P., PASSERON J.-C. (1970) : La reproduction, Paris : éd. de Minuit.

CORNU L. (2009) : "Normalité, normalisation, normativité : pour une pédagogie critique et inventive ", Le Télémaque, Presses Universitaires de Caen, n 36, p. 29-44.

DIOP C.-A. (1960) : L’Afrique noire pré-coloniale, Paris : Présence Africaine.

FOUCAULT M. (1976) : Histoire de la sexualité, T. 1, La volonté de savoir, Paris: Gallimard.

FORAY P. (2009) : "Trois formes de l'autorité scolaire», Le Télémaque, Presses universitaires de Caen, $n^{\circ} 35$, p. 73-86.

KAMBOUCHNER D. (2009) : "L'autorité pédagogique et la crise du sens des savoirs scolaires ", Le Télémaque, Presses universitaires de Caen, nº 35, p. 97-112.

REICHENBACH R. (2009) : "Domination masquée - Soumission douce : remarques sur les formes subtiles de l'autorité pédagogique ", Le Télémaque, n 35, p. 87-96.

SOLOMON TSEHAYE R. (2014): "La gestion des conflits culturels inhérents au choix éducatif à Djibouti : entre dénégation, défense et interculturation ", Éducation et sociétés, $\mathrm{n}^{\circ} 33, \mathrm{p} .79-95$.

UNICEF, Statistiques, Djibouti. Page consultée le 8 juin 2016 :

http://www.unicef.org/french/infobycountry/djibouti_statistics.html

VIEILLE-GROSJEAN H. (2016) : Culture(s) et Education(s), Conférence à l'Université de Fribourg, Bachelor de pédagogie clinique curative et d'éducation spécialisée. 